\title{
Rate of WD-WD head-on collisions in isolated triples is too low to explain standard type la supernovae
}

\author{
S. Toonen ${ }^{1,2,3}$, H. B. Perets ${ }^{2}$, and A. S. Hamers ${ }^{4,5}$ \\ 1 Anton Pannekoek Institute for Astronomy, University of Amsterdam, 1090 GE Amsterdam, The Netherlands \\ e-mail: toonen@uva.nl \\ 2 Department of Physics, Technion, 3200003 Haifa, Israel \\ 3 Departments of Physics and Astronomy, University of California, Berkeley, CA 94720, USA \\ ${ }^{4}$ Institute for Advanced Study, School of Natural Sciences, Einstein Drive, Princeton, NJ 08540, USA \\ ${ }^{5}$ Leiden Observatory, Leiden University, PO Box 9513, 2300 RA Leiden, The Netherlands
}

Received 1 September 2017 / Accepted 23 November 2017

\begin{abstract}
Context. Type Ia supernovae (Ia-SNe) are thought to arise from the thermonuclear explosions of white dwarfs (WDs). The progenitors of such explosions are still highly debated; in particular the conditions leading to detonations in WDs are not well understood in most of the suggested progenitor models. Nevertheless, direct head-on collisions of two WDs were shown to give rise to detonations and produce Ia-SNe - like explosions, and were suggested as possible progenitors.

Aims. The rates of such collisions in dense globular clusters are far below the observed rates of type Ia SNe, but it was suggested that quasi-secular evolution of hierarchical triples could produce a high rate of such collisions. With regular secular evolution, the expected Ia-SNe rate from isolated triples is orders of magnitude below the observed rate. Here we aim to test if the rate of WD collisions in triples can be significantly enhanced if quasi-secular evolution is taken into account.

Methods. We used detailed triple stellar evolution populations synthesis models coupled with dynamical secular evolution to calculate the rates of WD-WD collisions in triples and their properties. We explored a range of models with different realistic initial conditions and derived the expected SNe total mass, mass-ratio and delay time distributions for each of the models.

Results. We find that the SNe rate from WD-WD collisions is of the order of $0.1 \%$ of the observed Ia-SNe rate across all our models, and the delay-time distribution is almost uniform in time, and is inconsistent with observations.

Conclusions. We conclude that SNe from WD-WD collisions in isolated triples can at most provide for a small fraction of Ia-SNe, and can not serve as the main progenitors of such explosions.
\end{abstract}

Key words. binaries: close - stars: evolution - supernovae: general

\section{Introduction}

Type Ia supernovae (Ia-SNe) have played a pivotal role in our understanding of the structure of the universe and its rate of expansion through their use as standardizable candles (Phillips 1993; Riess et al. 1998; Perlmutter et al. 1999), as well as the chemical composition and evolution of galaxies (e.g. Pagel 1997). However, despite the significance, the origins of these $\mathrm{SNe}$ are still hotly debated (see e.g. Hillebrandt \& Niemeyer 2000; Maoz et al. 2014, for reviews).

Regular Ia-SNe (there are several classes of peculiar Ia-SNe, which we do not discuss here, see e.g. Li et al. 2001; Graur et al. 2017; Taubenberger 2017) are powered by the thermonuclear explosion of a carbon-oxygen (CO) white dwarf (WD), and several astrophysical scenarios leading to Ia-SNe explosions have been proposed, and one or even multiple progenitor channels may exist (Hillebrandt \& Niemeyer 2000; Maoz \& Mannucci 2012). There are three classical progenitor channels of which two concern a WD reaching the Chandrasekhar mass limit. This happens either by accretion from a non-degenerate companion star in the single-degenerate (SD) channel (Whelan \& Iben 1973; Nomoto 1982), or by a merger of two CO WDs in the double-degenerate (DD) channel (Iben \& Tutukov 1984; Webbink 1984). Another Chandrasekhar-mass channel, concerns the merger of a WD and an AGB-star degenerate core (Kashi \& Soker 2011; Ilkov \& Soker 2012; Soker 2013). Additionally, subChandrasekhar models have been considered through for example the double-detonation channel after He-accretion Woosley et al. (e.g. 1986), Livne (e.g. 1990). In recent years the DD scenario has been extended to include WD-WD collisions and not only mergers, but the former were thought to be extremely rare, and occur only in dense stellar clusters. For this reason they attracted relatively little attention compared with other WD explosion progenitors. Such collisions, however, are likely to be observable as type Ia SNe (Rosswog et al. 2009) and possibly non-standard SNe (Raskin et al. 2009, 2010; Papish \& Perets 2016). Recently, it was shown that some triple systems may dynamically evolve through a quasi-secular process, reminiscent of Kozai-Lidov oscillations (Kozai 1962; Lidov 1962), but where significant pericentre changes can occur on a single orbit timescale (Antonini \& Perets 2012), leading to extremely close pericentre approaches. In particular, it was suggested that such evolution in triples hosting an inner WD-WD binary could lead to physical collisions and the production of type Ia $\mathrm{SNe}$ (Katz \& Dong 2012; Thompson 2011; Kushnir et al. 2013).

The WD-WD collision scenario has several advantages. In particular, the detonation mechanism (shock ignition) is well understood and robust compared with other progenitor models 
(Kushnir et al. 2013), and the model may provide a range of Ia$\mathrm{SNe}$ with properties consistent with the observed ones. Physical collisions of WDs have been considered in the context of dense environments such as globular clusters and the Galactic centre (Hut \& Inagaki 1985; Sigurdsson \& Phinney 1993). However, the rates are expected to be several orders of magnitude below that of the observed Ia-SNe rate (Benz et al. 1989). The rate of collisions arising from a different channel, namely the evolution of isolated triples with quasi-secular evolution, however, was suggested to be high (Katz \& Dong 2012). Nevertheless, it was never self-consistently estimated and preliminary calculations suggested it is actually low (Hamers et al. 2013; Soker et al. 2014; Papish \& Perets 2016). In particular, Hamers et al. (2013) studied the SNIa rate from isolated triples with secular evolution theory. They focus on wide inner binaries that do not interact in the absence of the tertiary star. They find a rate that is low compared to observations $(\sim 0.1 \%)$, which predominantly comes from triples with wide inner binaries that experience mass transfer (their "circular mergers"). Here we try to close our knowledge gap and calculate self-consistently the expected rate of collisions due to quasi-secular evolution, test the viability of the isolated-triples WD-WD collision model (hereafter the WD-collision model) in term of the progenitor production rates, and derive the delay time distribution (DTD) and collision components (masses) in such explosions. As we show in the following, we find that the SNe rate from WD-WD collisions is of the order of at most $0.1 \%$ of the observed Ia-SNe rate across all the models we explored, and the delay-time distribution is almost uniform in time, and is inconsistent with observations and unlikely to explain the origins of standard Ia-SNe.

We begin by describing the triple population synthesis we use (Sect. 2) and the method applied to couple it with quasisecular triple evolution. We then lay out our assumptions and our criteria for identifying WD-WD collisions in our models and list the range of initial conditions explored. We then describe our detailed results for each of our models 3 and then discuss the results and summarize in Sect. 4.

\section{Method}

We studied the collision rate of WDs in triples by simulating the evolution of populations of triples. The first triple population synthesis studied were done in the context of destabilized triples due to stellar evolution (Perets \& Kratter 2012), which did not account for secular dynamics. Later Hamers et al. (2013) and Naoz et al. (2016) developed population synthesis codes, which included secular evolution, but do not account for quasi-secular regime. Here we used a recently developed triple population synthesis code TRES (Toonen et al. 2016; see Sect.2.2 for details) which we complement with a simplified treatment of the quasisecular evolution implications for collisions.

The simulation of a triple system starts with three stars on the zero-age main-sequence in a specific orbital configuration. As the distribution of masses and orbital parameters of these primordial triples is not well known, we applied different model populations to assess the systematic error on our calculations (Sect. 2.1).

The evolution from the main-sequence onwards is simulated with the triple evolution code TRES (Sect. 2.2). We considered triples that avoid mass transfer and remain dynamically stable throughout their evolution. Our triples evolve into a triple WD (3WD), or a double WD in the inner binary with a stellar tertiary (2WD). Generally, the tertiary has a low mass $\left(m_{3}<0.95 M_{\odot}\right)$ such that it does not evolve into a WD within a Hubble time. Subsequently, a collision occurs between the WDs in the inner binary that leads to the SNIa explosion. The collision can occur due to secular dynamics in three-body systems which drive the inner eccentricity to high values. However Katz \& Dong (2012) demonstrated the collision rate may be significantly enhanced for marginally hierarchical systems due to a breakdown of the secular approximation in the quasi-secular regime. We applied two methods to extract the systems that have evolved to a marginal hierarchy (Sect. 2.3). For these systems, the tertiary can significantly change the angular momentum of the inner binary by order unity during a pericentre passage. Katz \& Dong (2012) show that the angular momentum phase space is stochastically scanned, such that after a large number of pericentre passages a collision can be expected. Subsequently, the time to reach a collision $t_{\mathrm{col}}$ roughly follows a Poissonian distribution with a mean of:

$\tilde{t}_{\mathrm{col}}=\frac{a_{\mathrm{in}}}{4 R_{\mathrm{wd}}} P_{\mathrm{in}}$

where $P_{\text {in }}$ is the period of the inner binary, and $R_{\mathrm{wd}}$ is the radius of a WD here taken to be $10^{9} \mathrm{~cm}$. Throughout our analysis we assume that a triple entering into the quasi-secular regime leads to the inner binary direct collision on this given timescale.

\subsection{Primordial triples}

We performed simulations for six sets of primordial triples. These differ with respect to the distributions of stellar and orbital parameters (Table 1). In the standard model STD, we assume the mass $\left(m_{1}\right)$ of the initially most massive star in the inner binary (hereafter primary) follows the Kroupa initial mass function (IMF, Kroupa et al. 1993). The mass ratios of the inner binary ( $q_{\text {inner }} \equiv m_{2} / m_{1}$, where $m_{2}$ is the mass of the secondary) are distributed uniformly (Sana et al. 2012; Duchêne \& Kraus 2013; Moe \& Di Stefano 2017). We assumed that the mass $m_{3}$ of the outer companion (hereafter tertiary) is uncorrelated to that of the inner stars. This is consistent with observations of binaries with wide orbits (Moe \& Di Stefano 2017). Furthermore, in model STD the inner and outer semi-major axes $\left(a_{\text {inner }}\right.$ and $\left.a_{\text {outer }}\right)$ are distributed uniformly in log-space $(N \propto 1 / a)$, Abt (1983) between $5 \mathrm{R}_{\odot}$ and $5 \times 10^{6} R_{\odot}$. The eccentricities $e_{\text {inner }}$ and $e_{\text {outer }}$ are distributed thermally (Heggie 1975) between 0 and 1 . The mutual inclination follows a circular uniform distribution between 0 and $\pi$. We assumed that the arguments of pericentre and the lines of ascending nodes of both the inner and outer orbits are distributed uniformly between $-\pi$ and $\pi$. We assumed the stars have Solar metallicities. Finally, we adopted a constant binary fraction of $40 \%$ and triple fraction of $10 \%$ appropriate for Solar-mass stars (Raghavan et al. 2010; Duchêne \& Kraus 2013; Tokovinin 2014; Moe \& Di Stefano 2017). Systems that are dynamically unstable at initialization are rejected.

Our alternative models each differ from model STD in one aspect. In model Q_IN, we assumed that the masses of the inner binary are uncorrelated, as for the outer orbit of model STD. In model Q_OUT, we made the opposite assumption such that both the inner $q_{\text {inner }}$ and outer mass ratio $q_{\text {outer }} \equiv m_{3} /\left(m_{1}+m_{2}\right)$ are distributed uniformly. In model A_SANA, the distribution of the inner and outer semi-major axes follow a power-law distribution $N \propto(\log P)^{-0.55}$, as observed in binaries with $\mathrm{O}$ - and B-type primaries (Sana et al. 2012). For A-type primaries in binaries, Rizzuto et al. (2013) found a log-normal distribution of semi-major axes ( $\mu=0.95 \mathrm{AU}, \sigma=1.35)$, which we adopted in model A_RIZ with a maximum separation of $5 \times 10^{8} R_{\odot}$. Lastly, 
Table 1. Distributions of the initial binary masses and orbital parameters for the different models.

\begin{tabular}{l|cccccc}
\hline \hline Model & $q_{\text {inner }}$ & $q_{\text {outer }}$ & $a_{\text {inner }}$ & $a_{\text {outer }}$ & $e_{\text {inner }}$ & $e_{\text {outer }}$ \\
\hline STD & uniform & uncorrelated & log-uniform & log-uniform & thermal $^{2}$ & thermal \\
Q_IN & uncorrelated & uncorrelated & $\log$-uniform & $\log$-uniform & thermal & thermal \\
Q_OUT & uniform & uniform & $\log$-uniform & $\log$-uniform & thermal & thermal \\
A_SANA & uniform & uncorrelated & power-law $^{3}$ & power-law & thermal & thermal \\
A_RIZ & uniform & uncorrelated & log-normal $^{4}$ & log-normal & thermal & thermal \\
E_CIRC & uniform & uncorrelated & log-uniform & log-uniform & $\mathbf{0}$ & $\mathbf{0}$ \\
\hline
\end{tabular}

References. ${ }^{(1)}$ Abt (1983); (2) Heggie (1975); ${ }^{(3)}$ Sana et al. (2012); ${ }^{(4)}$ Rizzuto et al. (2013).

Table 2. Results of the population synthesis modelling.

\begin{tabular}{|c|c|c|c|c|c|c|c|c|c|c|}
\hline & \multirow[b]{2}{*}{ Method } & \multirow[b]{2}{*}{$N_{\text {sim }}$} & \multirow[b]{2}{*}{$f_{\text {param.space }}$} & \multicolumn{7}{|c|}{ Fraction } \\
\hline & & & & $\begin{array}{l}\text { mass } \\
\text { transfer }\end{array}$ & $\begin{array}{c}\text { dyn. } \\
\text { unstable }\end{array}$ & $\begin{array}{l}\text { quasi- } \\
\text { secular }\end{array}$ & $2 \mathrm{WD}$ & $\begin{array}{l}\text { 2WD quasi- } \\
\text { secular }\end{array}$ & $3 W D$ & $\begin{array}{l}\text { 3WD quasi- } \\
\text { secular }\end{array}$ \\
\hline \multirow[t]{2}{*}{ STD } & 1 & $50 \mathrm{k}$ & \multirow{2}{*}{$5.5 \times 10^{-3}$} & 0.093 & 0.11 & 0.018 & 0.70 & 0.0043 & 0.060 & $-(<2 \mathrm{e}-5)$ \\
\hline & 2 & $250 \mathrm{k}$ & & 0.099 & 0.11 & - & 0.70 & 0.0050 & 0.060 & $1.3 \times 10^{-4}$ \\
\hline \multirow[t]{2}{*}{ Q_IN } & 1 & $50 \mathrm{k}$ & \multirow{2}{*}{$9.7 \times 10^{-4}$} & 0.085 & 0.11 & 0.020 & 0.67 & 0.0039 & 0.060 & $2 \times 10^{-5}$ \\
\hline & 2 & $150 \mathrm{k}$ & & 0.095 & 0.11 & - & 0.67 & 0.0050 & 0.062 & $1.3 \times 10^{-4}$ \\
\hline \multirow[t]{2}{*}{ Q_OUT } & 1 & $50 \mathrm{k}$ & \multirow{2}{*}{$5.4 \times 10^{-3}$} & 0.10 & 0.095 & 0.046 & 0.19 & 0.0026 & 0.54 & $3.8 \times 10^{-4}$ \\
\hline & 2 & $100 \mathrm{k}$ & & 0.12 & 0.099 & - & 0.19 & 0.0019 & 0.54 & $1.6 \times 10^{-3}$ \\
\hline \multirow[t]{2}{*}{ A_SANA } & 1 & $50 \mathrm{k}$ & \multirow{2}{*}{$1.8 \times 10^{-3}$} & 0.11 & 0.11 & 0.015 & 0.68 & 0.0030 & 0.061 & $4 \times 10^{-5}$ \\
\hline & 2 & $150 \mathrm{k}$ & & 0.12 & 0.11 & - & 0.68 & 0.0043 & 0.062 & $9.4 \times 10^{-5}$ \\
\hline \multirow[t]{2}{*}{ A_RIZ } & 1 & $50 \mathrm{k}$ & \multirow{2}{*}{$3.0 \times 10^{-3}$} & 0.14 & 0.12 & 0.012 & 0.64 & 0.0022 & 0.056 & - \\
\hline & 2 & $100 \mathrm{k}$ & & 0.14 & 0.12 & - & 0.65 & 0.0028 & 0.055 & $3.0 \times 10^{-5}$ \\
\hline \multirow[t]{2}{*}{ E_CIRC } & 1 & $50 \mathrm{k}$ & \multirow{2}{*}{$1.0 \times 10^{-2}$} & 0.062 & 0.10 & 0.019 & 0.72 & 0.0045 & 0.067 & - \\
\hline & 2 & $15 \mathrm{k}$ & & 0.067 & 0.099 & - & 0.73 & 0.0069 & 0.068 & $2.8 \times 10^{-4}$ \\
\hline
\end{tabular}

in model E_CIRC we studied the effect of eccentricities on the collision rate. Initially these triples are circularized.

We considered stellar triples with $m_{1}>0.08 M_{\odot}$ and $m_{2}, m_{3}>0.008 M_{\odot}$. When drawing a mass from the Kroupa IMF, we adopted a maximum mass of $100 M_{\odot}$. To speed up the simulations, we only simulated a subset of triples (comprising a fraction $f_{\text {param.space }}$ of parameter space, see Table 2) that satisfy the following three requirements: 1) the inner binary can evolve into a double white dwarf within a Hubble time, that is $0.95 M_{\odot}<m_{1}, m_{2}<7.7 M_{\odot}$; 2) to avoid dissolution by the supernova $\left.m_{3}<7.7 M_{\odot} ; 3\right)$ to avoid mass transfer $a\left(1-e^{2}\right)>2500 R_{\odot}$. The latter is a conservative limit that excludes even isolated binaries, as tides would circularize the binary to the semi-latus rectum. We implicitly assume that the parameter space that we do not consider does not give rise to Ia$\mathrm{SNe}$ in the evolutionary channel considered here. The missing triples are taken into account in the normalization of the rates.

\subsection{Simulating triple evolution with TRES}

TRES is an astrophysical code to simulate the evolution of stellar triples consistently (Toonen et al. 2016); the code couples three-body dynamics with stellar evolution including KozaiLidov oscillations, tides, gravitational wave emission, and the effects of precession and stellar winds. The dynamics is based on the secular approach up to and including octupole-order (e.g. Naoz 2016, for a review). Stellar evolution is simulated in a parametrized way through the binary population synthesis code SeBa (Portegies Zwart \& Verbunt 1996; Toonen et al. 2012). TRES is valid for simulating isolated coeval stellar triples. Moreover, due to the usage of the secular approach, it is strictly only appropriate to simulate the evolution of hierarchical triples.
TRES is written in the Astrophysics Multipurpose Software Environment, or AMUSE (Portegies Zwart et al. 2009, 2013). It is a software framework that includes codes from different astrophysical domains, such as stellar dynamics, stellar evolution, hydrodynamics and radiative transfer. AMUSE provides the user with a homogeneous interface structure based on Python in which the community codes can be easily used and coupled. AMUSE can be downloaded for free at amusecode.org and github.com/amusecode/amuse.

\subsection{Marginal hierarchical systems}

We applied two methods to extract those triples that become marginally hierarchical during their evolution. In method 1 , we tracked the level of hierarchy in the simulations with TRES. When a system enters the "quasi-secular" regime, the simulation is stopped ${ }^{1}$, according to the following boundary condition we implemented in TRES:

$$
\sqrt{1-e_{1}}<\sqrt{1-e_{\text {crit }}} \equiv f_{\text {crit }} \times 5 \pi \frac{m_{3}}{m_{1}+m_{2}}\left[\frac{a_{1}}{a_{2}\left(1-e_{2}\right)}\right]^{3}
$$

1 We note that after a system enters the quasi-secular regime, it may still take a long time before the collision occurs (see Eq. (1)). If the triple is a $2 \mathrm{WD}$ with a relatively massive stellar tertiary, stellar evolution may still play a role for the evolution of the system. For all 2 WDs in the quasi-secular regime $\leqslant 10 \%$ have a tertiary star with mass above $0.95 M_{\odot}$. An exception to this is model Q_OUT, in which $63 \%$ of quasi-secular 2 WDs have a massive tertiary that will evolve of the mainsequence in a Hubble time. The treatment of the quasi-secular regime in this case is therefore not self-consistent. A better modelling is beyond the scope of this project and could be explored in the future. 
where $f_{\text {crit }}$ is a numerical factor $\approx 1$ (Antonini et al. 2014; see also Antonini \& Perets 2012; Katz \& Dong 2012). If the inner eccentricity becomes larger than $e_{\text {crit }}$, the angular momentum of the inner orbit can change by order of itself in one period. We note that with this criterion, the SNIa progenitors in our models predominantly fulfill the criterion (see Eq. (7) in Katz \& Dong 2012) to experience a clean collision (see also Perets \& Kratter 2012 for a similar clean collision criterion); all pericentre passages before the collision are large enough such that tidal or general relativistic effects are negligible. Cases where tidal interactions occur are treated by the regular secular evolution coupled to tidal evolution.

In method 2, the simulations with TRES were performed until time $t_{\text {form }}$, which represents the time at which the triple WD forms, or the double WD if the tertiary star does not evolve to a WD in a Hubble time.

We calculated analytically the maximum eccentricity $e_{\max }$ that the inner orbit can achieve afterwards based on the quadrupole approximation for $e_{\text {in }} \geq 0$ (e.g. Kinoshita \& Nakai 2007; Perets \& Naoz 2009; Naoz 2016). If $e_{\max }>e_{\text {crit }}$ (Eq. (2)), we assumed the system enters the quasi-secular regime. Such a simplified criterion does not well capture the maximal eccentricity potentially reached due to octupole-level perturbations, however the latter typically become important for inner binaries with low-mass ratio, while WD-WD binaries in our study always have a high mass ratio (typically above 0.85 ). If a triple enters the quasi-secular regime before $t_{\text {form }}$, the evolution of the system is evaluated based on the secular approach, which is not strictly valid in this regime. However, it is likely that the system reaches high eccentricities even within the secular approximation, such that Roche lobe overflow develops. Mass transferring systems are not taken into account in our rate estimates. Method 2 is less accurate than method 1 , but allows for more flexibility by varying $f$.

\section{Results}

\subsection{Formation}

In order to calculate the rate of colliding WDs, we first study the formation rate of a DWD with an outer companion, either another WD (i.e. 3WD) or a low-mass stellar component (i.e. 2WD). In most of our models, about $6 \%$ of primordial triples evolve to a $3 \mathrm{WD}$ in a Hubble time, and about $70 \%$ to a 2WD (Table 2). An exception to this is model Q_OUT, in which the masses of the outer companion are correlated to those of the inner binaries and the average tertiary mass is therefore higher compared with the other models. In model Q_OUT 3WDs are formed more efficiently; about half of the primordial triples evolve to a $3 \mathrm{WD}$ and a fifth evolve to become a $2 \mathrm{WD}$.

About $25 \%$ of triples do not become a $2 \mathrm{WD}$ or $3 \mathrm{WD}$ (Table 2). Mainly, these systems become dynamically unstable due to their stellar and orbital evolution or the systems experience mass transfer. Regarding the former, triples that become dynamically unstable due to mass loss in the stellar winds are studied by Perets \& Kratter (2012). This evolutionary channel can lead to a stellar collision involving an (post-)AGB star. Perets \& Kratter (2012) find that this channel could be the dominant form of stellar collisions in the field. Regarding the latter, double WDs that form through mass transfer are expected to have gone through a significant reduction of their orbit during the prior mass transfer phase between the primary WD and the hydrogen-rich progenitor of the secondary (Nelemans et al. 2000; van der Sluys et al. 2006). Assuming that the orbit of the tertiary does not shrink as well (a modest widening is expected if the mass loss from the inner binary acts as a fast non-interacting wind), the hierarchy of the triple has increased due to the mass transfer phase. This would make it less likely for triples with mass transfer to experience a collision in the inner double white dwarf due to quasi-secular evolution. In this paper we exclude triples that experience mass transfer. We note that overall the merger rate between WDs is enhanced if the binary has a tertiary companion, and some direct collisions may occur even outside the quasi-secular regime. Indeed, non-quasi-secular mergers and collisions in triples were studied by Hamers et al. (2013), however, such mergers and collisions do not contribute more than $10^{-3}$ of the Ia rate at any given time.

Next we consider those systems that become marginally hierarchical. In method 1, we track the level of hierarchy at every timestep in the simulations with TRES. We find that a few percent of all simulated triples reach sufficiently high inner eccentricities such that the systems enter the quasi-secular regime. The fraction is highest in model Q_OUT, which is related to the high average tertiary masses in this model. Of interest here are the 2WDs and 3 WDs that become quasi-secular. All six models show that even though the formation of a $2 \mathrm{WD}$ or $3 \mathrm{WD}$ is common (in the part of parameter space simulated here), only a small percentage of the triples reach this state, that is $0.2-0.5 \%$. In method 2, we find similar percentages of $0.2-0.7 \%$.

\subsection{Supernovae rates and delay time distributions}

We find that head-on collisions between carbon-oxygen WDs from wide isolated triples happen at a rate of a few times $10^{-7}$ per solar mass of created stars. The rates of the different models and methods are given in Table 3. In comparison, the observed rate of supernova Type Ia in field galaxies is about $10^{-3} M_{\odot}^{-1}$ (e.g. Maoz et al. 2014; Maoz \& Graur 2017) and therefore the contribution the isolated-triples channel to the SNIa rate is of the order of $0.01-0.1 \%$.

The different models of primordial triples give rise to up to an order of magnitude uncertainty in the synthetic Ia-SNe rates. The highest rates are expected if the inner and outer orbits are circularized (model E_CIRC). In this case fewer systems will undergo mass transfer, as indicated in Table 2 by the large fraction of parameter space that is simulated $f_{\text {param.space, }}$ and the small fraction of systems experiencing mass transfer in the simulated triples. Consequently, these systems will follow a different evolutionary channel than considered here. The lowest rates are anticipated if the masses of the three stars are not correlated to one another (model Q_IN). In this case the average mass of the secondary is low, its evolutionary timescale is long, such that fewer triples will harbour two WDs in the inner binary (see $f_{\text {param.space }}$ in Table 2). Furthermore, as the average mass of the tertiary is low, the dynamical effect of the tertiary on the inner binary is smaller, and fewer systems enter the quasi-secular regime.

Another aspect that affects the predicted collision rate is the extent of the quasi-secular regime. So far we have adopted a sharp boundary between the secular and quasi-secular regime, that is $f_{\text {crit }}=1$. In reality, the reliability of the secular approximation deteriorates gradually when approaching the critical boundary. Assuming that the secular approximation falls short at $f_{\text {crit }}=2\left(f_{\text {crit }}=10\right)$ and using method 2 , the collision rate increases by about a factor $\sim 2-3(\sim 10-40)$.

The collision rate depends also on the abundance of triples, that is the triple fraction. Here we have assumed a triple fraction of $10 \%$ and a binary fraction of $40 \%$. These values 
Table 3. Time-integrated collision rate of CO-CO WDs per solar mass of created stars.

\begin{tabular}{l|ll}
\hline \hline Model & Method 1 & Method 2 \\
\hline STD & $3.0 \times 10^{-7}$ & $4.7 \times 10^{-7}$ \\
Q_IN & $4.7 \times 10^{-8}$ & $7.5 \times 10^{-8}$ \\
Q_OUT & $1.5 \times 10^{-7}$ & $2.6 \times 10^{-7}$ \\
A_SANA & $1.0 \times 10^{-7}$ & $2.0 \times 10^{-7}$ \\
A_RIZ & $2.4 \times 10^{-7}$ & $4.3 \times 10^{-7}$ \\
E_CIRC & $6.1 \times 10^{-7}$ & $1.7 \times 10^{-6}$ \\
\hline Observed $^{1}$ & \multicolumn{2}{|l}{$(1.3 \pm 0.1) \times 10^{-3},(1.6 \pm 0.3) \times 10^{-3}$} \\
\hline
\end{tabular}

Notes. The different models are described in Sect.2.1, and the methods in Sect.2.

References. (1) Maoz \& Graur (2017), Maoz et al. (2014).

Table 4. Delay time distribution at long delay times in field galaxies.

\begin{tabular}{ccc}
\hline \hline Delay (Gyr) & DTD & Reference \\
\hline $8.1_{-5.7}^{+5.7}$ & $3_{-0.6}^{+1.5}$ & Maoz et al. (2011) \\
$8.1_{-5.7}^{+5.7}$ & $1.8_{-0.4}^{+0.4}$ & Maoz et al. (2012) \\
$8.1_{-5.7}^{+5.7}$ & $4.5_{-0.6,-0.5}^{+0.6+0.3}$ & Graur \& Maoz (2013) \\
\hline
\end{tabular}

Notes. Taken from Maoz \& Graur (2017).

are based on observation of Solar-type stars (Raghavan et al. 2010; Duchêne \& Kraus 2013; Tokovinin 2008, 2014). However, several studies have shown that the binary fraction varies with the stellar type of the primary (Raghavan et al. 2010; Duchêne \& Kraus 2013; Klein \& Katz 2017; Moe \& Di Stefano 2017). The binary fraction increases with primary mass, and there are indications that the triple fraction follows a similar trend (Remage Evans 2011; Sana et al. 2014; Moe \& Di Stefano 2017). Assuming a triple fraction of $25 \%$ and a binary fraction of $60 \%$ (appropriate for A-type stars), the collision rates in Table 3 increase by a factor of approximately two. Taking the most optimistic and likely not realistic assumptions, that is a high triple fraction, $f_{\text {crit }}=10$ and model E_CIRC one can reach a level of $\sim 6 \%$ of Ia-SNe from this channel. A more plausible fraction would of the order of $0.1-1 \%$ of Ia-SNe.

The collision rate as a function of time since a single burst of star formation, that is the delay time distribution (DTD) is shown in Figs. 1 and 2. These shown DTDs are for all models in method 2. The predicted DTDs from method 1 (not shown) are in good agreement with those shown in the figures. We find that the DTDs of all models and methods have a very distinct shape, namely the delay times are distributed uniformly in time. On the other hand, the observed SNIa DTD decreases strongly with time. The characteristic shape of the observed DTD is $\mathrm{d} N / \mathrm{d} t \propto t^{\beta}$ with $\beta \approx-1$ (Graur et al. 2011; Graur \& Maoz 2013; Heringer et al. 2017).

Due to the shape of the predicted and observed DTD, the largest contribution from head-on collisions in isolated triples is expected at long delay times. At these times, the observed SNIa rate in field galaxies is about $10^{-14} \mathrm{yr}^{-1} M_{\odot}^{-1}$ (Table 4), and our maximum predicted collision rate is about $10^{-16} \mathrm{yr}^{-1} M_{\odot}^{-1}$ (model E_CIRC). At best, collisions in triples contribute about $1 \%$ to the SNIa rate at late times.

\subsection{Masses and mass-ratios of colliding WDs}

Now we turn to the masses of the colliding WDs. The combined mass of the colliding WDs and their mass ratios are shown

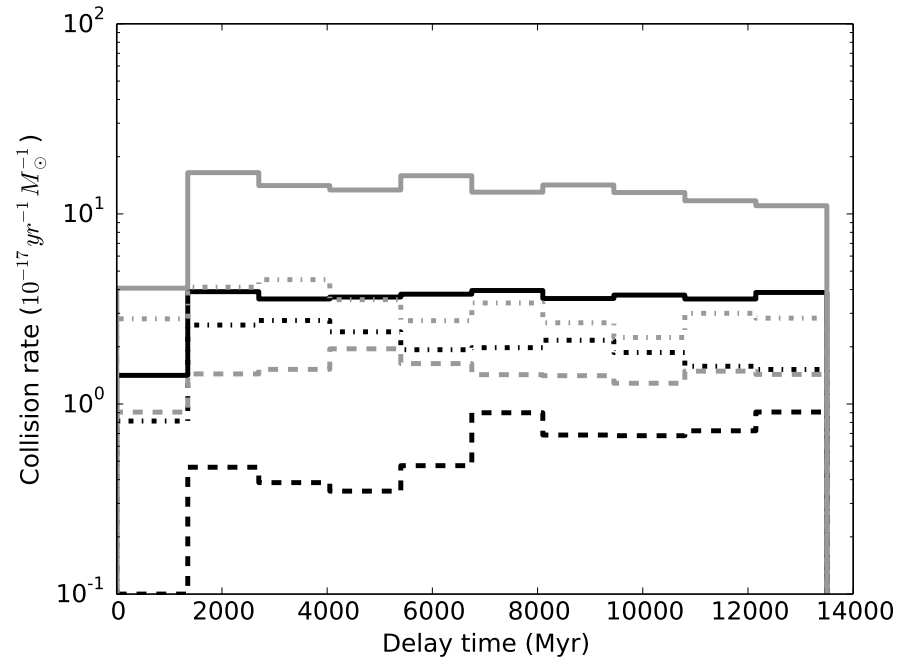

Fig. 1. Delay time distribution of head-on collisions between white dwarfs in isolated triples. The rate of collisions is given per $10^{17} \mathrm{yr}$ per solar mass of created stars. The different line-styles correspond to the different models in method 2. The DTD is approximately uniform in time. Both the normalization and the shape of the DTD is in clear contradiction with observations.

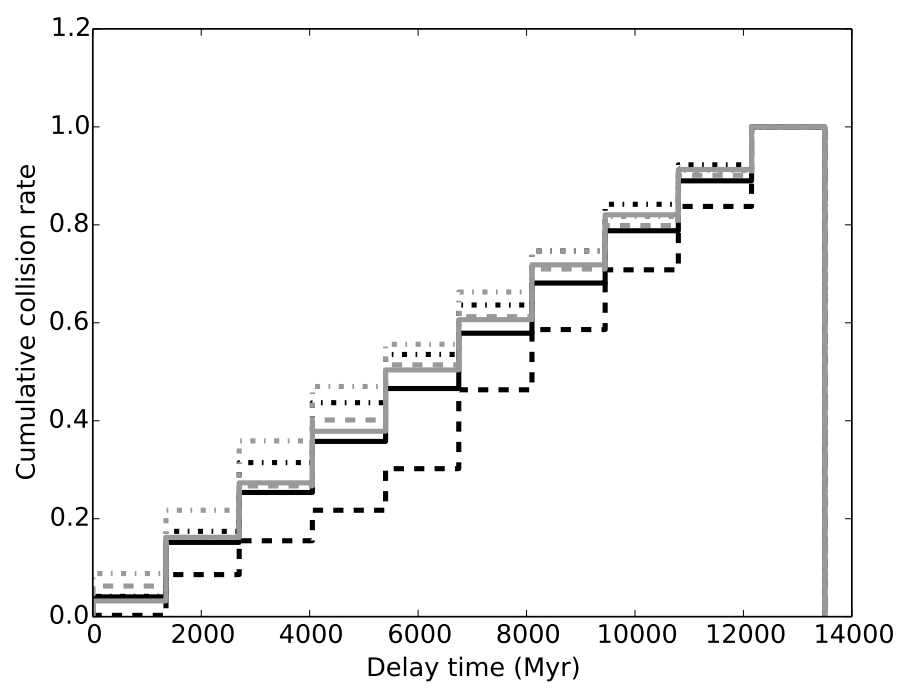

Fig. 2. Cumulative delay time distribution of head-on collisions between white dwarfs in isolated triples. The collision rates are normalized to 1 .

in Figs. 3 and 4. These figures represent model STD using method 2, but all models show similar behaviours. The stellar evolution timescales are long for (single) low-mass stars that is low-mass WD progenitors. Therefore WD-WD systems with high total mass tend to form earlier, and therefore collide following a short delay time, while low-mass systems form later and thereby give rise to long delay times.

The individual masses of the colliding WDs are close to one another (Fig. 4). This is expected as the initial-to-final mass relation for WDs is fairly flat, for example Kalirai et al. (2008) who find $M_{\text {final }}=(0.109 \pm 0.007) M_{\text {initial }}\left(M_{\odot}\right)+(0.394 \pm 0.025) M_{\odot}$.

The masses of the colliding WDs are important for the amount of ${ }^{56} \mathrm{Ni}$ that can be synthesized in the collision (see e.g. García-Senz et al. 2013). Early works of hydrodynamical simulations of colliding WDs have found a minimum mass ratio $(q \gtrsim 0.6-0.7)$ and/or total mass $\gtrsim 1.0-1.2 M_{\odot}$ necessary for an explosion to take place. On the other hand, at higher resolution 


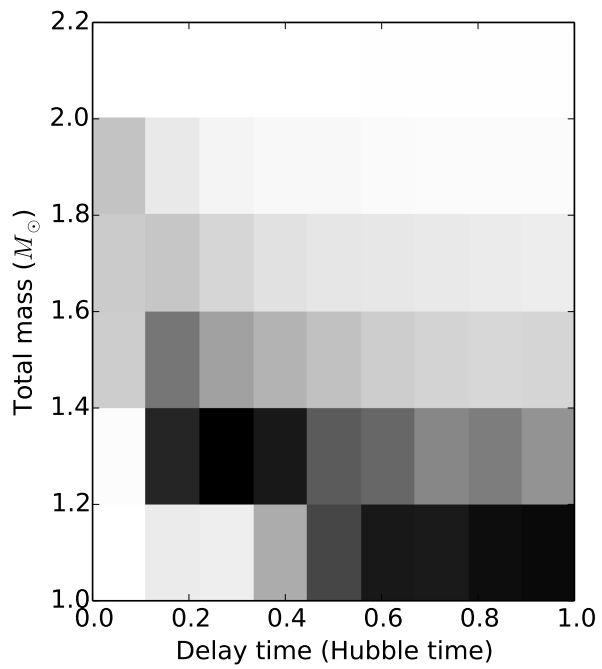

Fig. 3. Total mass of the colliding white dwarfs of the inner binary as a function of the delay time for model STD and method 2. Delay time is given as a fraction of the Hubble time, here taken as 13.5 Gyr. The grey scale is a density of objects on a linear scale.

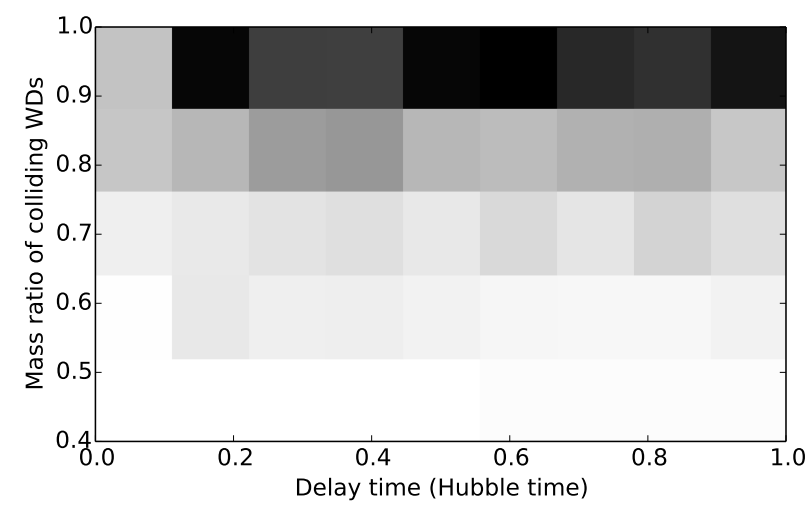

Fig. 4. Mass ratio of the colliding white dwarfs of the inner binary as a function of the delay time for model STD and method 2. Delay time is given as a fraction of the Hubble time, here taken as 13.5 Gyr. The grey scale is a density of objects on a linear scale.

Kushnir et al. (2013) found all their collisions to produce enough ${ }^{56} \mathrm{Ni}$ in order to appear as a SNIa. In our simulations, the mass ratios are typically $\sim 0.9-1$ (Fig. 4 ), such that a minimum mass ratio of $\sim 0.6-0.7$ does not effect the predicted collision rate significantly. On the other hand if the minimum total mass of the colliding WDs to produce a SNIa-like event is $1.2 M_{\odot}$, the synthetic rates given in this paper can be seen as an upper limit (Fig. 3).

Lastly, Piro et al. (2014) compared the distribution of ${ }^{56} \mathrm{Ni}$ derived from SNIa observations with the expected yields from colliding WDs (based on Kushnir et al. 2013). Piro et al. (2014) find that if colliding WDs are the main channel to form SNIa, the average mass of the WDs should be peaked around $0.75 M_{\odot}$. This is not reproduced by our models.

\section{Discussion and summary}

In this paper we studied the rate of WD-WD direct collisions induced by secular and quasi-secular evolution in triple stellar systems, and their potential to explain the origin of typical type Ia SNe. Though triple secular evolution was shown to produce only low-rates of direct WD-WD collisions (Hamers et al. 2013), it was suggested that less hierarchical triple systems can evolve through quasi-secular evolution (Antonini \& Perets 2012) leading to high rates of WD-WD collisions in triple (Katz \& Dong 2012). In order to study this possibility we used a novel triple population synthesis models TRES, coupled to simplistic application of quasi-secular evolution, when relevant, and explored the properties of such triple-formed SNe, including their rates, total mass of the colliding WDs, the WDs mass-ratios and the expected delay time distribution. Given the many uncertainties in the properties and quasi-secular evolution of triple systems, we constructed a range of plausible models for the triple progenitor population, and employed several simplified models to account for the quasi-secular evolution involved. Though the predicted rates may range over an order of magnitude, depending on the chosen model, all models predict no more than $\sim 0.1 \%$ of regular type Ia may arise from WD collisions in isolated triples. Moreover, the delay time distribution of SNe from this channel is distributed uniformly over time, and is therefore inconsistent with that inferred from observations $\left(\sim t^{-1}\right)$.

Many of the potential progenitors that initially have high inclinations and relatively weak hierarchy, that "active" triples susceptible to the quasi-secular evolution already dynamically evolve into mass-transfer, mergers or collisions during the mainsequence of giant-branch stages, and never produce WD-WD binaries for which collisions can be induced by the third stellar companion. Possible channels to introduce more active triples with WD-WD inner binaries in the relevant phase space could be through the perturbations of "non-active" triple, such as a triple with low-mutual inclination which would not quasi-secularly evolve significantly otherwise. In stellar clusters triples could be perturbed by other stars in the cluster and thereby change their orbital parameters. The evolution of such non-isolated perturbed triples is not considered here, however; the total number of triples in clusters is relatively small, both due to the total number of stars in clusters in general in addition to the small fraction of triples, which would need to be sufficiently compact ("hard") as not to be disrupted by encounters with other stars. We therefore do not expect triples in cluster to contribute significantly to the formation of type Ia SNe.

Triples in the field might also be susceptible to flyby encounters by field stars. However, such flybys introduce negligible changes in the triple orbits, and at most minor changes in the orbits of the widest triples. Study of flybys in very wide triples will be explored elsewhere, but these too are not expected to contribute significantly. We conclude that triples, and in particular isolated field triples, are likely to produce only a small fraction (at most a percent) of type Ia SNe.

Acknowledgements. S.T. gratefully acknowledges support from the Netherlands Research Council NWO (grant VENI [No. 639.041.645]). H.B.P. and S.T. gratefully acknowledge support from the Israel science foundation I-CORE program 1829/12. A.S.H. gratefully acknowledges support from the Institute for Advanced Study, and from NASA grant NNX14AM24G.

\section{References}

Abt, H. A. 1983, ARA\&A, 21, 343

Antonini, F., Murray, N., \& Mikkola, S. 2014, ApJ, 781, 45

Antonini, F., \& Perets, H. B. 2012, ApJ, 757, 27

Benz, W., Thielemann, F.-K., \& Hills, J. G. 1989, ApJ, 342, 986

Duchêne, G., \& Kraus, A. 2013, ARA\&A, 51, 269

García-Senz, D., Cabezón, R. M., Arcones, A., Relaño, A., \& Thielemann, F. K. 2013, MNRAS, 436, 3413

Graur, O., \& Maoz, D. 2013, MNRAS, 430, 1746

Graur, O., Poznanski, D., Maoz, D., et al. 2011, MNRAS, 417, 916

Graur, O., Bianco, F. B., Modjaz, M., et al. 2017, ApJ, 837, 121 
Hamers, A. S., Pols, O. R., Claeys, J. S. W., \& Nelemans, G. 2013, MNRAS, 430, 2262

Heggie, D. C. 1975, MNRAS, 173, 729

Heringer, E., Pritchet, C., Kezwer, J., et al. 2017, ApJ, 834, 15

Hillebrandt, W., \& Niemeyer, J. C. 2000, ARA\&A, 38, 191

Hut, P., \& Inagaki, S. 1985, ApJ, 298, 502

Iben, Jr., I., \& Tutukov, A. V. 1984, ApJS, 54, 335

Ilkov, M., \& Soker, N. 2012, MNRAS, 419, 1695

Kalirai, J. S., Hansen, B. M. S., Kelson, D. D., et al. 2008, ApJ, 676, 594

Kashi, A., \& Soker, N. 2011, MNRAS, 417, 1466

Katz, B., \& Dong, S. 2012, ArXiv e-prints [arXiv: 1211.4584]

Kinoshita, H., \& Nakai, H. 2007, Celes. Mech. Dyn. Astron., 98, 67

Klein, Y. Y., \& Katz, B. 2017, MNRAS, 465, L44

Kozai, Y. 1962, AJ, 67, 591

Kroupa, P., Tout, C. A., \& Gilmore, G. 1993, MNRAS, 262, 545

Kushnir, D., Katz, B., Dong, S., Livne, E., \& Fernández, R. 2013, ApJ, 778, L37

Li, W., Filippenko, A. V., Treffers, R. R., et al. 2001, ApJ, 546, 734

Lidov, M. L. 1962, Planet. Space Sci., 9, 719

Livne, E. 1990, ApJ, 354, L53

Maoz, D., \& Graur, O. 2017, ApJ, 848, 25

Maoz, D., \& Mannucci, F. 2012, PASA, 29, 447

Maoz, D., Mannucci, F., Li, W., et al. 2011, MNRAS, 412, 1508

Maoz, D., Mannucci, F., \& Brandt, T. D. 2012, MNRAS, 426, 3282

Maoz, D., Mannucci, F., \& Nelemans, G. 2014, ARA\&A, 52, 107

Moe, M., \& Di Stefano, R. 2017, ApJS, 230, 15

Naoz, S. 2016, ARA\&A, 54, 441

Naoz, S., Fragos, T., Geller, A., Stephan, A. P., \& Rasio, F. A. 2016, ApJ, 822, L24

Nelemans, G., Verbunt, F., Yungelson, L. R., \& Portegies Zwart, S. F. 2000, A\&A, 360, 1011

Nomoto, K. 1982, ApJ, 253, 798

Pagel, B. E. J. 1997, Nucleosynthesis and Chemical Evolution of Galaxies (Cambridge, UK: Cambridge University Press)

Papish, O., \& Perets, H. B. 2016, ApJ, 822, 19

Perets, H. B., \& Kratter, K. M. 2012, ApJ, 760, 99
Perets, H. B., \& Naoz, S. 2009, ApJ, 699, L17

Perlmutter, S., Aldering, G., Goldhaber, G., et al. 1999, ApJ, 517, 565

Phillips, M. M. 1993, ApJ, 413, L105

Piro, A. L., Thompson, T. A., \& Kochanek, C. S. 2014, MNRAS, 438, 3456

Portegies Zwart, S., McMillan, S., Harfst, S., et al. 2009, New A, 14, 369

Portegies Zwart, S., McMillan, S. L. W., van Elteren, E., Pelupessy, I., \& de Vries, N. 2013, Comput. Phys. Commun., 183, 456

Portegies Zwart, S. F., \& Verbunt, F. 1996, A\&A, 309, 179

Raghavan, D., McAlister, H. A., Henry, T. J., et al. 2010, ApJS, 190, 1

Raskin, C., Timmes, F. X., Scannapieco, E., Diehl, S., \& Fryer, C. 2009, MNRAS, 399, L156

Raskin, C., Scannapieco, E., Rockefeller, G., et al. 2010, ApJ, 724, 111

Remage Evans, N. 2011, Bulletin de la Societe Royale des Sciences de Liege, 80,663

Riess, A. G., Filippenko, A. V., Challis, P., et al. 1998, AJ, 116, 1009

Rizzuto, A. C., Ireland, M. J., Robertson, J. G., et al. 2013, MNRAS, 436, 1694

Rosswog, S., Kasen, D., Guillochon, J., \& Ramirez-Ruiz, E. 2009, ApJ, 705, L128

Sana, H., de Mink, S. E., de Koter, A., et al. 2012, Science, 337, 444

Sana, H., Le Bouquin, J.-B., Lacour, S., et al. 2014, ApJS, 215, 15

Sigurdsson, S., \& Phinney, E. S. 1993, ApJ, 415, 631

Soker, N. 2013, in Binary Paths to Type Ia Supernovae Explosions, eds. R. Di Stefano, M. Orio, \& M. Moe, IAU Symp., 281, 72

Soker, N., García-Berro, E., \& Althaus, L. G. 2014, MNRAS, 437, L66

Taubenberger, S. 2017, ArXiv e-prints [arXiv: 1703.00528]

Thompson, T. A. 2011, ApJ, 741, 82

Tokovinin, A. 2008, MNRAS, 389, 925

Tokovinin, A. 2014, AJ, 147, 87

Toonen, S., Hamers, A., \& Portegies Zwart, S. 2016, Comput. Astrophys. Cosmol., 3, 6

Toonen, S., Nelemans, G., \& Portegies Zwart, S. 2012, A\&A, 546, A70

van der Sluys, M. V., Verbunt, F., \& Pols, O. R. 2006, A\&A, 460, 209

Webbink, R. F. 1984, ApJ, 277, 355

Whelan, J., \& Iben, Jr., I. 1973, ApJ, 186, 1007

Woosley, S. E., Taam, R. E., \& Weaver, T. A. 1986, ApJ, 301, 601 